\title{
Fibrosis endomiocárdica: una cardiomiopatía restrictiva en países en vía de desarrollo
}

\author{
Endomyocardial fibrosis: a restrictive cardiomyopathy in developing countries
}

\author{
Juan F. Vásquez-Rodríguez ${ }^{1 *}$, Ramón I. Medina-Mur ${ }^{1}$, Luis E. Giraldo ${ }^{1,2}$, Marietta L. Juan-Guardela ${ }^{1,2}$, \\ Julián Gelves ${ }^{1,3}$ y Claudia P. Jaimes ${ }^{1,3}$ \\ ${ }^{1}$ División de Cardiología, Fundación Cardiolnfantil IC, Universidad El Bosque, Facultad de Medicina y Ciencias de la salud; ${ }^{2}$ Universidad El Rosario, \\ Escuela de Medicina y Ciencias de la Salud; ${ }^{3}$ Departamento de Imagen Cardiovascular, Fundación Cardiolnfantil IC. Bogotá D.C., Colombia
}

\begin{abstract}
Resumen
Objetivo: La fibrosis endomiocárdica (FE) es una cardiomiopatía restrictiva infrecuente. En América Latina son escasos los reportes. En el presente trabajo se realiza una descripción de una serie de pacientes diagnosticados de FE en Colombia. Método: Realizamos una búsqueda en los registros de imágenes de resonancia magnética (RM) cardiaca realizadas en nuestra institución entre 2016 y 2019 en busca de pacientes con diagnóstico de FE. Se describieron sus características sociodemográficas, clínicas y de imagen. Resultados: Nueve pacientes fueron diagnosticados de FE (el $66.7 \%$ mujeres), con una edad promedio de 69 años. Los pacientes presentaron un promedio de 2.6 años de evolución. El principal síntoma referido fue disnea, seguido de síncope, dolor torácico y palpitaciones. En ninguno de ellos se sospechó FE como diagnóstico inicial. En cuanto a los hallazgos ecocardiográficos, se identificó compromiso predominante del ventrículo izquierdo, seguido de compromiso biventricular. Todos los pacientes presentaron patrón de llenado restrictivo con dilatación auricular izquierda severa. En el análisis retrospectivo se cumplieron los criterios de Mocumbi para el diagnóstico de FE en el 100\% de los pacientes con gravedad moderada (77.8\%). Las imágenes de RM mostraron función sistólica biventricular y volúmenes preservados. Se observó depósito focal de gadolinio subendocárdico a nivel apical y se confirmó la presencia de trombo en el 66\% de los casos. Conclusión: La FE es una cardiomiopatía restrictiva infrecuente circunscrita a países tropicales. La mayoría de los pacientes en nuestra serie presentaron compromiso aislado del ventrículo izquierdo, seguido de compromiso biventricular, con función ventricular usualmente preservada.
\end{abstract}

Palabras clave: Cardiomiopatía restrictiva. Falla cardiaca congestiva. Fibrosis endomiocárdica.

\begin{abstract}
Objective: Endomyocardial fibrosis (EF) is an unusual restrictive cardiomyopathy. In Latin America there are few reports. Here, we made a description of patients diagnosed with EF in Colombia. Method: We conducted a search in the records of cardiac magnetic resonance imaging (MRI) performed in our institution between 2016-2019 looking for patients with a diagnosis of EF; sociodemographic, clinical and imaging characteristics were described. Results: Nine patients were diagnosed with EF (66.7\% female), with an average age of 69 years. Patients presented an average evolution of 2.6 years. The main reported symptom was dyspnea, followed by syncope, chest pain, and palpitations. None of them was initially suspected for EF. Regarding echocardiographic findings, predominant left ventricular involvement was identified, followed by bi-ventricular

\section{Correspondencia:}

*Juan F. Vásquez-Rodríguez

E-mail: juanfevasquez8@gmail.com

Disponible en internet: 12-04-2021 Arch Cardiol Mex. 2021;91(2):196-201 www.archivoscardiologia.com 1405-9940 / @ 2020 Instituto Nacional de Cardiología Ignacio Chávez. Publicado por Permanyer. Este es un artículo open access bajo la licencia CC BY-NC-ND (http://creativecommons.org/licenses/by-nc-nd/4.0/).
\end{abstract}


involvement. All the patients presented a restrictive filling pattern with severe left atrial dilation. In a retrospective analysis, Mocumbi criteria for diagnosis of EF were met in $100 \%$ of the patients, majority with moderate severity (77.8\%). Cardiac MRI showed biventricular systolic function and volumes preserved. Focal subendocardial late gadolinium enhancement was observed on the apex and apical thrombus was confirmed in $66 \%$ of the patients. Conclusion: FE is an uncommon restrictive cardiomyopathy limited to tropical countries. Most of patients in our series presented isolated involvement of left ventricle, followed by bi-ventricular involvement, with ventricular function usually preserved.

Key words: Restrictive cardiomyopathy. Congestive heart failure. Endomyocardial fibrosis.

\section{Introducción}

La fibrosis endomiocárdica (FE) es una miocardiopatía restrictiva infrecuente considerada una enfermedad tropical asociada a países de «bajos ingresos». Al realizar una búsqueda en Medline con la palabra clave "endomyocardial fibrosis" se despliega una limitada fuente de información entre los años 1946 y 2020, con un promedio de 46 artículos por año. En 1948, Davis describió el primer caso en Uganda ${ }^{1}, y$ en la actualidad se estiman 10-12 millones de personas afectadas en todo el mundo ${ }^{2}$. La edad de presentación tiene un comportamiento bimodal, con un primer pico a los 10 años y un segundo pico a los 30 años $^{3}$. Los países con mayor prevalencia de la enfermedad se ubican en la línea ecuatorial, y Nigeria es el país más afectado.

En América Latina son escasos los reportes ${ }^{2}$ y la evidencia existente proviene de Brasil y Colombia; en este último país hay tres reportes de caso ${ }^{4-6}$ con características similares a las descritas en pacientes del continente africano.

El objetivo del presente trabajo es describir una serie de casos diagnosticados de FE en un centro de referencia cardiovascular en Bogotá, Colombia, señalando sus características sociodemográficas, clínicas y de diagnóstico por imagen cardiovascular multimodal.

\section{Método}

Realizamos una búsqueda en los registros de imágenes de resonancia magnética (RM) cardiaca llevadas a cabo en nuestra institución entre 2016 y 2019 en busca de pacientes con diagnóstico de FE. Los pacientes debían contar además con estudios de ecocardiografía realizados en la misma institución.

Los estudios de ecocardiografía fueron realizados con equipos Phillips iE33 (Koninklijke Philips n.v.), Phillips EPIQ7 (Koninklijke Philips n.v.) y GE VividE9 (General Electric Company). Las imágenes de RM cardiaca se obtuvieron con un equipo Phillips Ingenia 1.5 $\mathrm{T}$ (Koninklijke Philips n.v.).
Se describieron las características sociodemográficas, clínicas y de imagen.

\section{Resultados}

Nueve pacientes fueron identificados con diagnóstico de FE. Las características generales se describen en la tabla 1 . El $66.7 \%$ fueron mujeres y la edad promedio fue de 69 años. El tiempo promedio entre el diagnóstico y la fecha de corte de recolección de datos fue de 2.6 años, sin registro de mortalidad en este periodo. El principal síntoma referido fue disnea, seguido de síncope, dolor torácico y palpitaciones. En los pacientes con síncope $(5,55,6 \%)$, la etiología fue clasificada de la siguiente manera: de origen cardiaco en dos por presencia de taquicardia ventricular no sostenida y disfunción sinusal diagnosticados por hallazgos en Holter de ritmo de 24 horas asociados a síntomas; neuromediado o vasovagal en dos pacientes con prueba de mesa basculante positiva con respuesta mixta; y ortostático en un paciente con posible disfunción autonómica por diabetes y altas dosis de antihipertensivos.

En todos los pacientes se contó con al menos un trazo electrocardiográfico de 12 derivaciones. Seis de los nueve pacientes se encontraban en ritmo sinusal y los tres restantes en ritmo de fibrilación auricular con respuesta ventricular controlada. De los seis pacientes en ritmo sinusal, todos presentaron algún trastorno de la conducción intraventricular, cinco de ellos caracterizados como trastorno inespecífico y uno como bloqueo completo de rama izquierda. Todos los pacientes presentaron trastornos difusos de la repolarización relacionados con los trastornos de conducción descritos previamente.

En ninguno de ellos se sospechó FE como diagnóstico clínico inicial. La sospecha diagnóstica se suscitó después del estudio ecocardiográfico, sin realizar un diagnóstico definitivo en ninguno de los casos por este método.

El ecocardiograma identificó compromiso predominante del ventrículo izquierdo, seguido por compromiso 
Tabla 1. Características generales de la población

\begin{tabular}{|c|c|}
\hline & Total: 9 n (\%) \\
\hline Sexo femenino & $6(66.7)$ \\
\hline Edad (años) & $69 \pm 8$ \\
\hline $\begin{array}{l}\text { Síntomas } \\
\text { Dolor torácico } \\
\text { Disnea } \\
\text { Palpitaciones } \\
\text { Síncope } \\
\text { Edema de extremidades }\end{array}$ & $\begin{array}{l}3(33.3) \\
6(66.7) \\
3(33.3) \\
5(55.6) \\
1(11.1)\end{array}$ \\
\hline $\begin{array}{l}\text { Diagnóstico inicial } \\
\text { ICC } \\
\text { CMH } \\
\text { Cardiopatía isquémica } \\
\text { Trombo apical } \\
\text { Dolor torácico a estudio }\end{array}$ & $\begin{array}{l}3(33.3) \\
2(22.2) \\
2(22.2) \\
1(11.1) \\
1(11.1)\end{array}$ \\
\hline $\begin{array}{l}\text { Hallazgos ecocardiográficos } \\
\text { Compromiso biventricular } \\
\text { Compromiso ventrículo izquierdo } \\
\text { Compromiso ventrículo derecho }\end{array}$ & $\begin{array}{c}2(22.2) \\
7(77.8) \\
0(0)\end{array}$ \\
\hline FEVI (\%) & $45 \pm 11$ \\
\hline LVEDV (ml) & $58 \pm 15$ \\
\hline Volumen $\mathrm{Al}$ indexado $\left(\mathrm{ml} / \mathrm{m}^{2}\right)$ & $60 \pm 23$ \\
\hline Patrón restrictivo en diástole & $9(100)$ \\
\hline $\begin{array}{l}\text { Criterios diagnósticos de Mocumbi } \\
\text { Sí } \\
\text { Leve } \\
\text { Moderado } \\
\text { Grave }\end{array}$ & $\begin{array}{l}9(100) \\
1(11.1) \\
7(77.8) \\
1(11.1)\end{array}$ \\
\hline $\begin{array}{l}\text { Hallazgos RM cardiaca } \\
\text { FEVI }(\%) \\
\text { FEVD }(\%) \\
\text { LVEDV indexado }\left(\mathrm{ml} / \mathrm{m}^{2}\right) \\
\text { RVEDV indexado }\left(\mathrm{ml} / \mathrm{m}^{2}\right)\end{array}$ & $\begin{array}{c}60 \pm 4 \\
55 \pm 8.0 \\
59 \pm 25 \\
82 \pm 47\end{array}$ \\
\hline $\begin{array}{l}\text { Trombo apical (confirmado con RM cardiaca) } \\
\text { Sí }\end{array}$ & $6(66.6)$ \\
\hline
\end{tabular}

Al: aurícula izquierda; CMH: cardiomiopatía hipertrófica; FEVD: fracción de eyección del ventrículo derecho; FEVI: fracción de eyección del ventrículo izquierdo; ICC: insuficiencia cardiaca congestiva; LVEDV: volumen de fin de diástole del ventrículo izquierdo; RVEDV: volumen de fin de diástole del ventrículo derecho; RM: resonancia magnética.

biventricular, con disfunción sistólica ventricular izquierda leve. Todos los pacientes presentaron patrón de llenado restrictivo con dilatación auricular izquierda grave. Los principales diagnósticos fueron cardiomiopatía hipertrófica o trombo apical (o ambos). Al realizar el análisis retrospectivo, el $100 \%$ de los pacientes cumplieron los criterios de Mocumbi (Tabla 2) para diagnóstico de FE, siendo de grado moderado en el $77.8 \%$.

De los parámetros evaluados por los criterios diagnósticos ecocardiográficos, los más frecuentes fueron, en su orden: placas endomiocárdicas $>2 \mathrm{~mm}$ de grosor (9/9), obliteración del ápex ventricular derecho o izquierdo (9/9, predominantemente el ápex ventricular izquierdo, en ocho pacientes), patrón de flujo restrictivo a través de las válvulas mitral o tricúspide (9/9), dilatación auricular con ventrículos de tamaño normal (4/9), engrosamiento difuso de la valva anterior mitral (2/9) y disfunción de las válvulas auriculoventriculares debido a adhesión del aparato valvular a la pared ventricular $(1 / 9$, con presencia de insuficiencia mitral moderada).

En todos los pacientes se realizó caracterización con RM cardiaca, y la función sistólica biventricular y los volúmenes fueron normales. En la secuencia de realce tardío, el compromiso fue exclusivo del subendocardio en los segmentos apicales; en dos de los pacientes hubo extensión a los segmentos medios del ventrículo izquierdo. Se identificó trombo apical en el $66 \%$ de los pacientes.

Los pacientes descritos continuaron seguimiento clínico por cardiología. Al momento del egreso hospitalario, el $88,8 \%$ recibía un betabloqueador, el $77,7 \%$ un inhibidor de la enzima convertidora de angiotensina (IECA) o un antagonista de los receptores de angiotensina II (ARA-II), el $22,2 \%$ furosemida y solo el $10 \%$ espironolactona o hidroclorotiazida.

Después de revisar los datos sociodemográficos, clínicos y de imagen, no fue posible establecer alguna relación causal para la enfermedad.

\section{Discusión}

La fibrosis endomiocárdica es una condición inusual particularmente limitada a países denominados «del Trópico" o de bajos recursos ${ }^{2}$, frecuentemente subdiagnosticada y con pobre información descrita, lo que dificulta su identificación y tratamiento.

Esta condición no tiene una edad de presentación claramente establecida. Se menciona que los síntomas suelen ser tardíos en el curso de la enfermedad. Algunos autores señalan edades tempranas, con un pico en la infancia y un segundo pico en la edad adulta? Sin embargo, en nuestro estudio las edades oscilaron entre la quinta y la séptima décadas de la vida.

Se especula que la FE puede tener tres fases: una de inflamación aguda, usualmente asintomática y rara vez detectada, en la que es probable identificar eosinofilia ${ }^{9}$; otra indeterminada o de transición; y finalmente una fase crónica en la que se hacen evidentes los signos y síntomas de falla cardiaca. Este 
Tabla 2. Criterios diagnósticos ecocardiográficos FE - Criterios de Mocumbi

\begin{tabular}{|l|c|}
\hline Criterio & Puntos \\
\hline Criterios mayores & 2 \\
\hline Placas endomiocárdicas $>2$ mm de grosor & 3 \\
Parches endomiocárdicos delgados (<1 mm) que afecten más de una pared ventricular & 4 \\
Obliteración del ápex ventricular derecho o izquierdo & 4 \\
Trombo o contraste espontáneo en ausencia de disfunción ventricular grave & 4 \\
Retracción del ápex ventricular derecho & $1-4+$ \\
Disfunción de las válvulas auriculoventriculares debido a adhesión del aparato valvular a la pared ventricular & \\
\hline Criterios menores & 1 \\
Parches endomiocárdicos delgados que afecten una pared ventricular & 2 \\
Patrón de flujo restrictivo a través de las válvulas mitral o tricúspide & 2 \\
Apertura diastólica de la válvula pulmonar & 1 \\
Engrosamiento difuso de la valva anterior mitral & 2 \\
Dilatación auricular con ventrículos de tamaño normal & 1 \\
Movimiento en M del tabique interventricular y pared posterior plana* & 1 \\
Aumento en la densidad de la banda moderadora y otras bandas intraventriculares & \\
\hline
\end{tabular}

Diagnóstico definitivo con 2 criterios mayores o 1 criterio mayor y 2 criterios menores

Score < 8 : leve, Moderado $8-15$ y Severo $>15$

AV: Auriculoventricular.

*El movimiento $\mathrm{M}$ del tabique interventricular se refiere a un patrón de movimiento observado en la ecocardiografía en modo $\mathrm{M}$, que se cree se debe a la obliteración o restricción del ápice ventricular izquierdo combinado con regurgitación mitral.

+ Clasificar según la severidad de la insuficiencia.

Adaptado de Mocumbi, et al. $2008^{8}$.

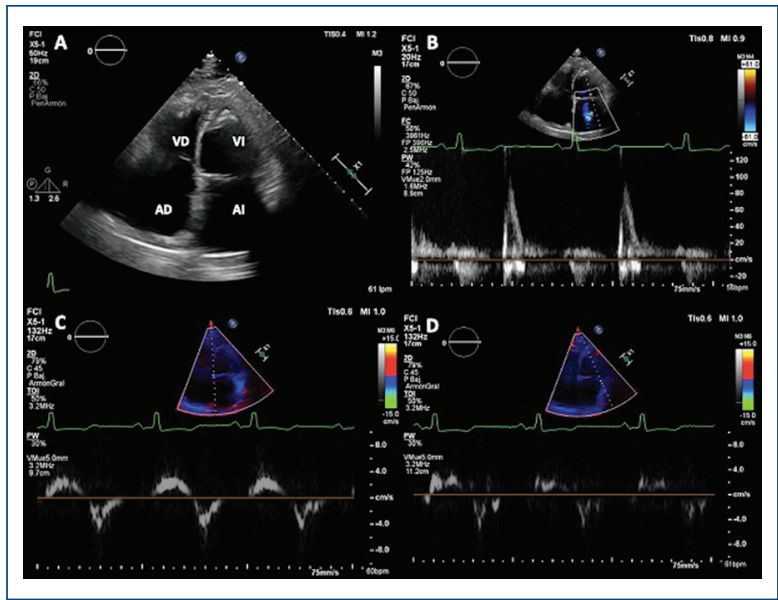

Figura 1. Hallazgos de FE en Ecocardiograma transtorácico. A: Proyección apical 4 cámaras con obliteración del ápex dilatación biauricular severa. B: Doppler pulsado de la válvula mitral con onda $E$ de $1.2 \mathrm{~m} / \mathrm{s}$, desaceleración < 160 msg y relación E/A > 2. C: Doppler tisular septal del anillo mitral, onda $e^{\prime}$ de $0.4 \mathrm{~m} / \mathrm{s}$. D: Doppler tisular lateral del anillo mitral, onda e' $0.4 \mathrm{~m} / \mathrm{s}$. Los hallazgos son concordante con disfunción diastólica restrictiva. AD: aurícula derecha; Al: aurícula izquierda; VD: ventrículo derecho; VI: ventrículo izquierdo.

comportamiento no ha podido ser confirmado en estudios clínicos².

La enfermedad se caracteriza por un patrón típicamente restrictivo (Fig. 1) secundario a un proceso de

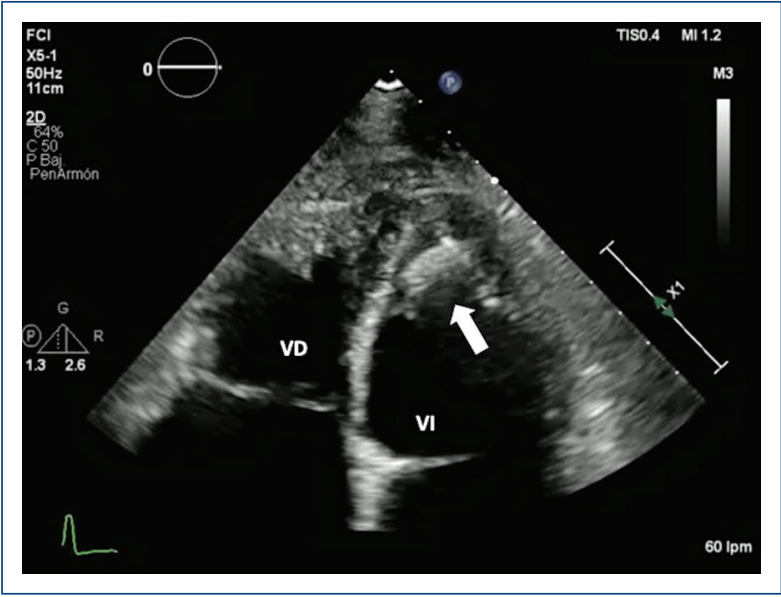

Figura 2. Ecocardiograma transtorácico. Proyección apical de cuatro cámaras con zoom para ventrículos, con obliteración apical y una imagen en el ápex sugestiva de trombo apical (flecha). VD: ventrículo derecho; VI: ventrículo izquierdo.

fibrosis que afecta la superficie subendocárdica, de localización típicamente apical (Fig. 2). Esta condición estuvo presente en todos nuestros pacientes.

Los estudios previos han descrito que el compromiso es biventricular en la mitad de los casos $(50 \%)$, seguido del compromiso exclusivo del ventrículo izquierdo (40\%) y del compromiso exclusivo del ventrículo derecho $(10 \%)^{10}$. En los pacientes con afección del ventrículo 


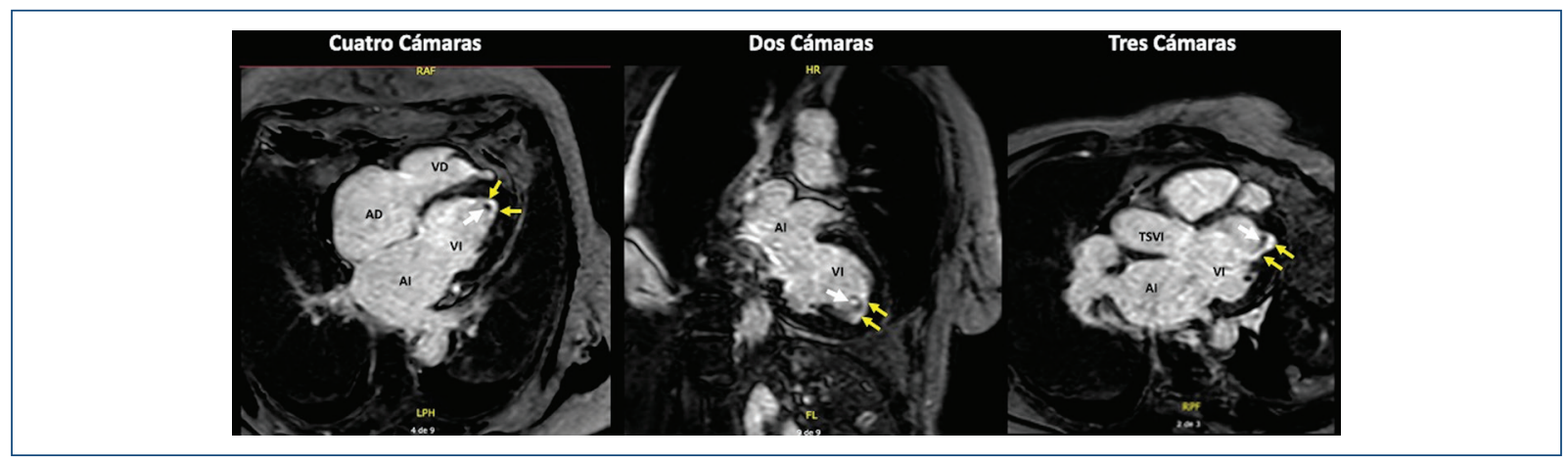

Figura 3. Resonancia nuclear magnética cardíaca, secuencias de realce tardío. Se aprecian zonas de depósito focal de gadolinio de distribución subendocárdica (flechas) que representan el compromiso por fibrosis endomiocárdica. Inmediatamente antes de las zonas de realce se aprecia una imagen ovoide hipointensa que corresponde con trombo apical. AD: aurícula derecha; Al: aurícula izquierda; TSVI: tracto de salida del ventrículo izquierdo; VD: ventrículo derecho; VI: ventrículo izquierdo.

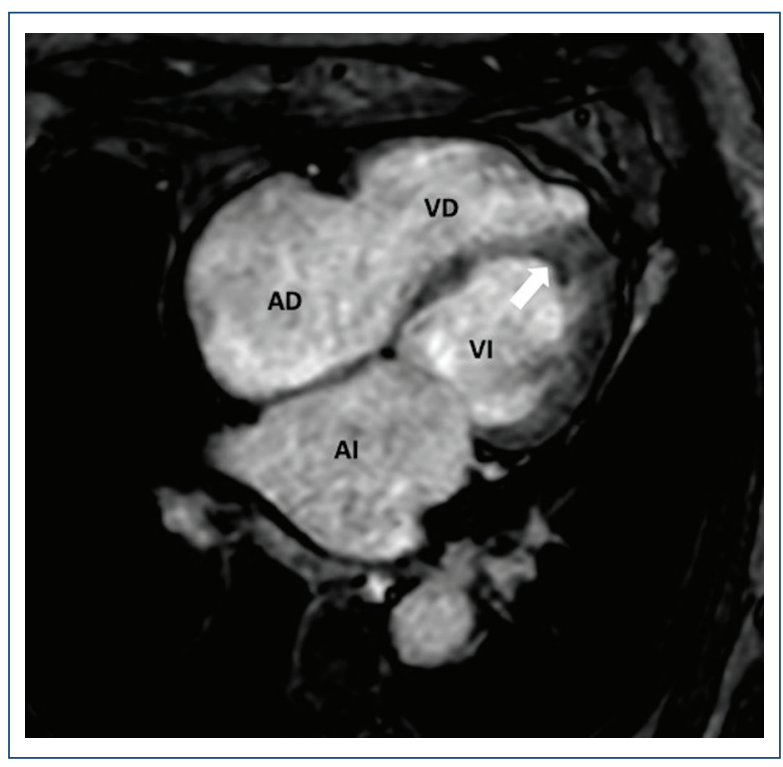

Figura 4. Resonancia nuclear magnética cardiaca de cuatro cámaras. Secuencia PSIR TFE, se aprecia imagen hipointensa (flecha correspondiente a trombo apical. AD: aurícula derecha; Al: aurícula izquierda; VD: ventrículo derecho; VI: ventrículo izquierdo.

izquierdo, la fibrosis puede extenderse a los segmentos medios y la valva posterior de la válvula mitral, respetando la valva anterior y el tracto de salida del ventrículo izquierdo ${ }^{11}$. En los pacientes aquí descritos, el compromiso ventricular izquierdo aislado fue el principal patrón de presentación, y solo hubo dos casos de compromiso biventricular y ninguno con afección exclusiva del ventrículo derecho.

Los pacientes con compromiso del ventrículo derecho presentan característicamente distensión yugular, hepatomegalia, ascitis y edema de los miembros inferiores $^{11}$. En algunos pacientes se ha identificado ascitis grave sin otros signos de falla cardiaca derecha, planteando la posibilidad de compromiso por fibrosis e inflamación del peritoneo ${ }^{2}$. No identificamos ascitis en nuestros pacientes.

Las teorías en cuanto a la etiología son diversas. En nuestra descripción no fue posible establecer relación causal alguna. Algunos agentes infecciosos, especialmente parasitarios como esquistosomas, filarias y plasmodios, se han relacionado con el desarrollo de la enfermedad, favoreciendo la presencia de eosinofilia y una posible reacción inflamatoria desmedida en el endocardio. También factores dietarios, como el consumo de cassava (Manihot esculenta crantz), tubérculo que contiene linamarina y que en el tracto gastrointestinal libera cianuro de hidrógeno, se han propuesto como otra posible etiología al alterar la biología del cardiomiocito. Los modelos animales han demostrado vacuolización intracelular y fibrosis intersticial del tejido miocárdico con el consumo de cassava ${ }^{3}$. Por último, se plantea una posible susceptibilidad genética, no muy bien sustentada hasta el momento.

El diagnóstico de la enfermedad es frecuentemente pasado por alto dado que se confunde con otras condiciones como la cardiomiopatía hipertrófica, la sarcoidosis cardiaca, la amiloidosis y trombos apicales ${ }^{12}$. En nuestra serie, los diagnósticos iniciales fueron, en su orden: insuficiencia cardiaca congestiva, cardiomiopatía hipertrófica, cardiopatía isquémica y trombo apical.

El ecocardiograma transtorácico es una herramienta de gran utilidad ante la sospecha de la 
enfermedad. En el año 2008 se realizó un estudio para evaluar la prevalencia de la enfermedad en una población rural de Mozambique empleando ecocardiografía transtorácica como método diagnóstico; de este derivan los criterios para el diagnóstico ecocardiográfico de $\mathrm{FE}$, también denominados criterios de Mocumbi (Tabla 2) ${ }^{8}$. El 100\% de la población analizada en nuestro trabajo cumplió los criterios diagnósticos, siendo los hallazgos más frecuentes la presencia de placas endomiocárdicas que pueden obliterar el ápex ventricular, asociado a patrón restrictivo y compromiso de las válvulas auriculoventriculares con algún grado de disfunción de estas. Los criterios diagnósticos permiten además estadificar la gravedad de la enfermedad, la cual fue moderada en la mayoría de los casos presentados.

La RM cardiaca es otra técnica de imagen de gran utilidad en el diagnóstico de FE, pues permite identificar las áreas de fibrosis de distribución subendocárdica (Fig. 3) y reclasificar el tamaño y la función de las cámaras ventriculares, como sucedió en la presente serie, así como la identificación de trombos apicales, que se han descrito hasta en un $40 \%$ de la población ${ }^{13}$. En nuestra serie se identificaron en el $66 \%$ de los pacientes (Figs. 3 y 4 ).

En cuanto al tratamiento y el pronóstico de la FE, algunas series reportan una sobrevida no mayor de 2 años en estadios avanzados de la enfermedad ${ }^{14}$. Esta fue mayor en los pacientes de nuestra serie, que han logrado más de 2 años de evolución desde el momento del diagnóstico, aún sin registro de mortalidad.

El tratamiento se basa en los lineamientos del cuidado convencional de la falla cardiaca crónica. Para los pacientes aquí descritos, el uso de betabloqueadores, IECA o ARA-II fue superior al $75 \%$. La terapia definitiva es el trasplante cardiaco.

\section{Conclusión}

La FE es un tipo de cardiopatía restrictiva infrecuente, diagnosticada en países denominados «del Trópico", con compromiso biventricular o ventricular izquierdo exclusivo, pronóstico variable y etiología pobremente establecida. El tratamiento se basa en el bloqueo neurohumoral, y el trasplante de corazón es la terapia definitiva.

\section{Financiamiento}

Esta investigación no recibió ninguna subvención específica de agencias de financiación en los sectores público, comercial o sin fines de lucro.

\section{Conflicto de intereses}

Los autores declaran no tener ningún conflicto de intereses.

\section{Responsabilidades éticas}

Protección de personas y animales. Los autores declaran que para esta investigación no se han realizado experimentos en seres humanos ni en animales.

Confidencialidad de los datos. Los autores declaran que han seguido los protocolos de su centro de trabajo sobre la publicación de datos de pacientes.

Derecho a la privacidad y consentimiento informado. Los autores declaran que en este artículo no aparecen datos de pacientes.

\section{Bibliografía}

1. Connor DH, Somers K, Hutt MSR, Manion WC, D'Arbela PG. Endomyocardial fibrosis in Uganda (Davies' disease). 1. An epidemiologic, clinical, and pathologic study. Am Heart J. 1967;74:687-709.

2. Beaton A, Mocumbi AO. Diagnosis and management of endomyocardial fibrosis. Cardiol Clin. 2017;35:87-98.

3. Grimaldi A, Mocumbi AO, Freers J, Lachaud M, Mirabel M, Ferreira B Narayanan K, et al. Tropical Endomyocardial Fibrosis: Natural History, Challenges, and Perspectives. Circulation. 2016;133(24):2503-15.

4. Rodríguez-González MJ, Torres AM, Echeverría LE. Fibrosis endomiocárdica. Rev Colomb Cardiol. 2017;24:407.e1-5.

5. Villamil-Munévar A, Barbosa C, Ternera-Barrios A, Luis J, Olaya A. Fibrosis endomiocárdica. Rev. Colomb. Cardiol. 2017;24(1):59.

6. Uenishi EK. Reporte de un caso de endomiocardiofibrosis en Colombia. Falta revista. 2014;21:414-8.

7. Bukhman G, Ziegler J, Parry E. Endomyocardial fibrosis: still a mystery after 60 years. PLoS Negl Trop Dis. 2008;2:1-7.

8. Mocumbi AO, Ferreira MB, Sidi D, Yacoub MH. A population study of endomyocardial fibrosis in a rural area of Mozambique. N Engl J Med. 2008;359:43-9.

9. Andy JJ, Bishara FF, Soyinka OO. Relation of severe eosinophilia and microfilariasis to chronic African endomyocardial fibrosis. Br Heart J. 1981;45:672-80.

10. Bhatti K, Lopez-Mattei J. Endomyocardial fibrosis. StatPearls. 2020. (Consultado el 26 de enero de 2020.) Disponible en: http://www.ncbi.nlm. nih.gov/pubmed/30020665

11. Marangou J, Beaton A, Aliku TO, Nunes MCP, Kangaharan N, ReményiB. Echocardiography in indigenous populations and resource poor settings. Hear Lung Circ. 2019;28:1427-35.

12. Dato I. How to recognize endomyocardial fibrosis? J Cardiovasc Med. 2015;16:547-51.

13. Gulati GS, Sharma AK, Paliwal S, Seth S, Ramakrishnan S, Jagia P et al. Cardiac magnetic resonance in tropical endomyocardial fibrosis. $j$ Cardiovasc Magn Reson. 2011;13(S1):P270.

14. Bhatti K, Bandlamudi M, Lopez-Mattei J. Endomyocardial Fibrosis. [Updated 2020 Aug 12]. In: StatPearls [Internet]. Treasure Island (FL): StatPearls Publishing; 2021 Jan-. Available from: https://www.ncbi.nlm.nih. gov/books/NBK513293/. 